\title{
Are Anti-Inflammatory Cytokines Associated with Cognitive Impairment in Patients with Insomnia Comorbid with Depression? A Pilot Study
}

\author{
Shuo $\mathrm{He}^{\mathrm{I}}$ \\ $\mathrm{Xi}$-Xi Chen ${ }^{2}$ \\ Wei Gel \\ Shuai Yang $\mathbb{D}^{\prime}$ \\ Jun-Tao Chen (D) \\ Jing-Wen Niu' \\ Lan $\mathrm{Xia}^{3}$ \\ Gui-Hai Chen' \\ 'Department of Neurology (Sleep \\ Disorders), The Affiliated Chaohu \\ Hospital of Anhui Medical University, \\ Hefei (Chaohu), 238000, People's \\ Republic of China; ${ }^{2}$ Department of \\ Anesthesiology, The First Affiliated \\ Hospital of Anhui Medical University, \\ Hefei, 230022, People's Republic of \\ China; ${ }^{3}$ Department of Neurology, The \\ Second Affiliated Hospital of Anhui \\ Medical University, Hefei, 23060I, \\ People's Republic of China
}

Background: To distinguish insomnia comorbid with depression (ICD) from chronic insomnia disorder (CID) by exploring the relationship between serum levels of frequently overlooked anti-inflammatory cytokines and cognitive function.

Methods: A total of 42 ICD patients, 63 CID patients, and 42 healthy control subjects were enrolled in the study. The Pittsburgh Sleep Quality Index and Hamilton Depression Rating Scale were used to assess sleep quality and depression severity, respectively. The ChineseBeijing version of Montreal Cognitive Assessment scale (MoCA-C) and Nine-Box Maze Test (NBMT) were used to assess cognitive function. Serum levels of anti-inflammatory interleukins (IL-1RA, IL-4, IL-5, IL-10, IL-13, and IL-28A), transforming growth factor (TGF)- $\beta 1$, granulocyte-macrophage colony-stimulating factor, interferon- $\gamma$, and the chemokine regulated upon activation, normal $\mathrm{T}$ cell expressed and secreted (RANTES) were measured by enzyme-linked immunosorbent assay.

Results: The ICD group had significantly more errors in the spatial reference task $(\mathrm{H}=2.55$, $\left.\mathrm{P}_{\mathrm{s}}=0.03\right)$ and spatial working memory task $\left(\mathrm{H}=5.67, \mathrm{P}_{\mathrm{s}}<0.01\right)$ of the NBMT, as well as lower levels of IL-1RA $\left(\mathrm{H}=-2.85, \mathrm{P}_{\mathrm{s}}=0.01\right)$, IL-4 $\left(\mathrm{H}=-3.28, \mathrm{P}_{\mathrm{s}}<0.01\right)$, IL-5 $\left(\mathrm{H}=-3.35, \mathrm{P}_{\mathrm{s}}<0.01\right)$, IL-10 $\left(\mathrm{H}=-4.46, \mathrm{P}_{\mathrm{s}}<0.01\right)$, and IL-28A $\left(\mathrm{H}=-2.75, \mathrm{P}_{\mathrm{s}}=0.02\right)$ than control subjects. Compared with the CID group, the ICD group had significantly more errors in the spatial reference memory task $\left(\mathrm{H}=-2.84, \mathrm{P}_{\mathrm{s}}=0.01\right)$ of the NBMT, and lower levels of IL-5 $\left(\mathrm{H}=3.41, \mathrm{P}_{\mathrm{s}}<0.01\right)$, IL-10 $\left(\mathrm{H}=5.30, \mathrm{P}_{\mathrm{s}}<0.01\right)$, IL-13 $\left(\mathrm{H}=3.89, \mathrm{P}_{\mathrm{s}}<0.01\right)$, and GM-CSF $\left(\mathrm{H}=2.72, \mathrm{P}_{\mathrm{s}}=0.02\right)$. A partial correlation analysis showed that the level of one or more of IL-4, IL-5, IL-10, IL-13, and TGF- $\beta 1$ was positively correlated with cognitive function (MoCA-C score and/or performance in spatial memory task) in ICD patients.

Conclusion: ICD is a distinct condition that can be distinguished from CID based on immune dysfunction and specific types of cognitive dysfunction.

Keywords: insomnia, depression, cytokine, cognition

\section{Introduction}

Depression is a chronic mental disorder that frequently co-occurs with other medical conditions or psychiatric illnesses; it has a high prevalence and is associated with high rates of recurrence and disability. ${ }^{1}$ Although depression typically manifests as low mood and cognitive impairment, chronic insomnia disorder (CID) is a frequent complaint. ${ }^{2,3}$ Insomnia is not only a risk factor for depression but is also a residual symptom following its treatment, ${ }^{4,5}$ highlighting a bidirectional relationship between insomnia and depression. ${ }^{6,7}$ Cognitive deficits are present in 
both disorders and exacerbate cognitive impairment in patients with depression. ${ }^{8-10}$ In the Diagnostic and Statistical Manual of Mental Disorders, Fifth Edition (DSM-5) and the International Classification of Sleep Disorders, Third Edition (ICSD-3), insomnia comorbid with other mental disorders must be excluded and diagnosed separately from CID. ${ }^{11,12}$

Inflammation has been proposed as a mechanism underlying multiple chronic diseases including depression, insomnia, and mild cognitive impairment. ${ }^{13-19}$ Among patients who received antidepressant treatment, patients with a low level of inflammation had better prognosis than those with a high level. ${ }^{20}$ Depression is thought to be related to activation of the immune response leading to the increased secretion of cytokines, ${ }^{21}$ which can cause cognitive impairment including changes in attention, learning, and memory. ${ }^{22}$ Additionally, inflammation plays an important role in sleep regulation and disturbance. Sleep promotes immune homeostasis through the modulation of inflammatory mediators such as cytokines, which regulate normal sleep-wake behavior. ${ }^{23}$ Insomnia is associated with several chronic infectious and inflammatory diseases. ${ }^{24}$ Thus, inflammation links depression, insomnia, and cognitive impairment, although it is unclear whether insomnia comorbid with depression (ICD) has a distinct immunologic profile.

Cytokines are polypeptides produced by immune cells that regulate cellular functions; they can be divided into six types according to their source-namely, interleukins (ILs), granulocyte-macrophage colony-stimulating factor (GM-CSF), interferon (IFN), tumor necrosis factor (TNF), growth factor, and chemokines such as regulated upon activation, normal $\mathrm{T}$ cell expressed and secreted (RANTES). ${ }^{25}$ There are two functional subtypes of cytokine: proinflammatory (IL-1, IL-1 $\beta$, IL-2, IL-6, IFN- $\gamma$, TNF- $\alpha$, TNF- $\beta$, etc) and anti-inflammatory (IL-1RA, IL4, IL-5, IL-10, IL-13, etc), which promote and inhibit immune activation, respectively. ${ }^{26-29}$ Although the cytokine profile of depression has not been clearly established, patients with depression exhibit cytokine abnormalities such as elevated levels of IL-1 $\beta$, IL-6, and TNF. ${ }^{30-32}$ Cognitive dysfunction is associated with elevated plasma levels of C-reactive protein and IL- $6 .{ }^{22,33}$ In addition, IL-1 and TNF are known to modulate sleep, while sleep deficiency induces immune system changes. ${ }^{23}$ Thus, while proinflammatory cytokines have been widely studied in the contexts of sleep disorder and depression, the roles of other inflammatory factors such as TGF, GM-CSF, IFN, and RANTES remain unclear.

The aims of this study were to 1) examine the correlation between immunologic profile and cognitive function in ICD and 2) distinguish ICD from CID by investigating the roles of the abovementioned cytokines (antiinflammatory ILs, TGF, GM-CSF, IFN, and RANTES) and cognitive function in ICD and CID.

\section{Methods}

\section{Subjects}

A total of 42 ICD patients were recruited continuously at the Clinic of Sleep and Memory Disorder of the Affiliated Chaohu Hospital of Anhui Medical University between January 2019 and April 2020. The flowchart of the study participants is presented in Figure 1. Besides simultaneously meeting ICSD-3 and DSM-5 diagnostic criteria for ICD, inclusion criteria for patients were as follows: 1) age between 18 and 75 years; 2) at least 9 years of education without problems in comprehension; 3) not taking antidepressants or other drugs that could potentially interfere with sleep, cognitive function, or endocrine function in the 3 months prior to enrollment; 4) Pittsburgh Sleep Quality Index (PSQI) $>7 ;^{34-36}$ 5) 17-Item Hamilton Depression Rating Scale (HAMD-17) score $\geq 7 ; ;^{37}$ and 6) participating voluntarily in the study after signing the informed consent form. The exclusion criteria were as follows: 1) any other somatic comorbidity (including immunologic, endocrine, cardiovascular, neurologic, liver, or kidney disease or organic brain disease); 2) history of substance abuse; 3) recent infection or inflammation (within 2 weeks of the start of the study); 4) taking drugs that could affect sleep, mood, immune function, and cognition; 5) shift-work subjects; and 6) pregnant or lactating women.

The study also enrolled 63 CID patients (insomnia lasting $\geq 6$ months; HAMD-17 score $<17$; no other disease comorbid with insomnia) and 42 control subjects (PSQI and HAMD-17 scores $<7$; no insomnia or related medical history during the same period). All the enrolled patients were the first to visit who did not take antidepressants or other drugs that could potentially interfere with sleep, cognitive function, or endocrine function in the 3 months prior to enrollment. The study was approved by the Affiliated Chaohu Hospital of Anhui Medical University Ethics Committee (approval no. 201805-kyxm-01). 


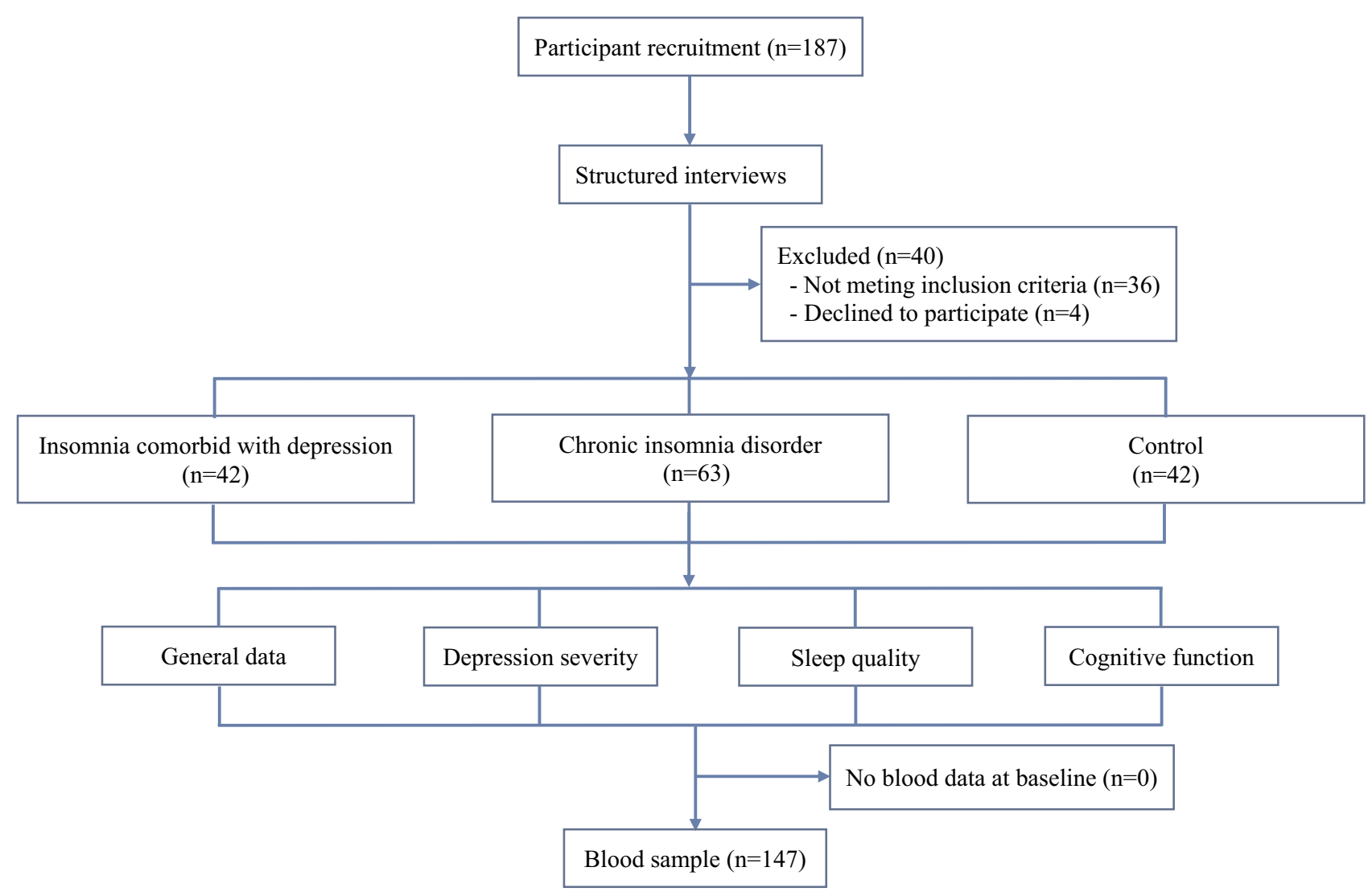

Figure I Flowchart of the study participants.

\section{General Data Collection}

General information was collected on each participant including sex, age, education level, body mass index (BMI), individual history, daily life history, medical history, and family medical history.

\section{Assessment of Depression Severity}

To ensure the consistency of enrolled patients, the Chinese version of Mini International Neuropsychiatry Interview 5.0 (Mini 5.0) was independently conducted by Master's- and doctoral-level evaluators with background and training in psychology. ${ }^{38}$ Depression severity was assessed with the HAMD-17, which comprises 17 items relating to depressed mood, feelings of guilt and suicide, sleep, work, and activities. ${ }^{37} \mathrm{~A}$ score $<7$ indicates a normal state while scores of 7-17, 18-24, and $>24$ correspond to mild, moderate, and severe depression, respectively.

\section{Evaluation of Sleep Quality}

Sleep quality was assessed with the PSQI, which has seven components including subjective sleep quality, sleep latency, sleep duration, habitual sleep efficiency, sleep disturbance, use of sleep medication, and daytime dysfunction during the previous month scored on a 4-point rating scale ranging from 0 (none) to 3 ( $\geq 3$ times per week). ${ }^{34}$ In China, a score $\geq 7$ has high diagnostic sensitivity and specificity for distinguishing patients with poor sleep from normal subjects. ${ }^{36}$ Total PSQI scores range from 0 to 21, with a higher score corresponding to worse sleep quality. ${ }^{34}$

\section{Cognitive Assessment}

The Chinese-Beijing Version of the Montreal Cognitive Assessment scale (MoCA-C) is a widely used cognitive screening test with good reliability and validity. ${ }^{39}$ The MoCA-C has eight dimensions: visual space and executive function, naming, attention, language, abstraction, shortterm memory, delayed recall, and orientation. ${ }^{40}$ The maximum score is 30 points, and a score $\geq 26$ indicates normal cognitive function. ${ }^{39}$

The Nine-Box Maze Test (NBMT), which was originally designed to assess spatial memory, was modified to 
evaluate multiple aspects of memory function including spatial/object working memory (SWM/OWM), spatial/ object reference memory (SRM/ORM), and object recognition memory (ORcM). ${ }^{41,42}$ The experiment was conducted according to our previous study. ${ }^{43}$ The test comprises object familiarization, training, and testing phases, and the final result is represented by the number of errors. A higher number of errors indicated poor memory.

\section{Serum Cytokine Profiling}

After the intervention and all other assessments had been completed, a 3-mL sample of blood was obtained from each subject the next morning between 7:30 a.m. and 8:00 a.m., with fasting and avoidance of strenuous activity and mental stimulation. On this condition, all serum cytokines measured showed stable values according to each individual's condition. The sample was allowed to stand at room temperature for $30 \mathrm{~min}$ and then centrifuged at $3000 \mathrm{rpm}$ for $5 \mathrm{~min}$; the serum was stored with anticoagulant and frozen at $-80^{\circ} \mathrm{C}$. TGF- $\beta 1$, GM-CSF, IFN- $\gamma$, and RANTES levels in the serum were measured using enzyme-linked immunosorbent assay kits (Shanghai Jianglai Co, Shanghai, China) and a microplate reader (Labsystems Multiskan MS 352; Thermo Fisher Scientific, Waltham, MA, USA), with a sensitivity $>84 \%$ and specificity $>98 \%$. All samples were prepared in duplicate and average values were used for analysis. Intra- and inter-assay coefficients of variation were $<9 \%$ and $<11 \%$, respectively.

\section{Statistical Analysis}

SPSS v.20 was used for statistical analyses. Continuous normally distributed data are presented as mean $\pm \mathrm{SD}$ and were evaluated with the Student's $t$-test to compare differences between groups and one-way analysis of variance to determine the main effects, with the least significant difference (LSD) test used for multiple comparisons. Nonnormally distributed data are expressed as $P 50(P 25, P 75)$, and differences between groups were analyzed with the rank-sum test for two independent samples with completely randomized design (Mann-Whitney $U$ ). The KruskalWallis $H$-test was used for multiple-group comparisons by adjusting the significance level to $\mathrm{P}<0.0167$ (Bonferroni correcting: $0.05 / 3$ ), and pairwise comparisons were conducted with the Student's $t$-test by manual calculation. Categorical data were analyzed with the chi-squared test. To control the influence of confounding factors to the variables, partial correlation analysis was used for the relationships among continuous variables. In partial correlation analysis, the confounder between serum cytokines and HAMD-17 (controlling for sex, age, education, BMI, and PSQI), serum cytokines and PSQI (controlling for sex, age, education, BMI, and HAMD-17), and serum cytokines and MoCA-C scores and spatial memory (controlling for sex, age, education, BMI, HAMD-17, and PSQI) are different. Two-sided $P$ values $\leq 0.05$ were considered statistically significant.

\section{Results}

\section{General Characteristics of the Study Subjects}

There were no significant differences in sex ratio $\left(c^{2}=2.7\right.$, $P=0.3)$, age $(\mathrm{F}=0.3, P=0.7)$, education level $(\mathrm{H}=3.1$, $P=0.2)$, or BMI $(\mathrm{t}=0.02, P=0.98)$ among the three groups (Table 1).

\section{Depression Severity}

HAMD scores of the three groups differed significantly $(\mathrm{H}=108.55, P<0.001)$ (Table 1). A pairwise comparison revealed that the ICD and CID groups had significantly higher scores than control subjects $\left(P_{s}<0.001\right)$. Additionally, the score for the ICD group was nearly two times higher than that of the CID group $(P<0.001)$.

\section{Sleep Quality}

PSQI scores differed significantly across groups $(\mathrm{H}=95.34$, $P<0.001)$. Both the ICD and CID groups had significantly higher scores than control subjects $\left(P_{S}<0.001\right)$, although there was no significant difference between them (Table 1).

\section{Cognitive Function}

There were no significant differences in MoCA scores among the 3 groups $(\mathrm{H}=1.85, P=0.40)$ (Table 1$)$. For performance in the NBMT, significant differences were observed in SRM $(\mathrm{H}=9.36, P=0.01)$, SWM $(\mathrm{H}=37.60$, $P<0.001)$, and $\mathrm{ORcM}(\mathrm{H}=8.79, P=0.01)$. The ICD group had more errors related to SRM than the CID and control groups ( $P=0.01 ; P=0.03)$, with no difference between the latter two groups $(P=1.00)$. The ICD and CID groups had more errors related to SWM than controls $\left(P_{s}<0.001\right)$ but there were no differences between them $(P=0.56)$. Only the CID group had significantly more errors related to ORcM than controls $(P=0.01)$ (Table 2$)$. 
Table I Demographic Characteristics, Depression Level, and Sleep Quality of the Study Subjects

\begin{tabular}{|c|c|c|c|c|c|c|c|c|}
\hline \multirow[t]{2}{*}{ Variable } & \multirow[t]{2}{*}{$\begin{array}{l}\text { Insomnia Comorbid } \\
\text { with Depression }\end{array}$} & \multirow{2}{*}{$\begin{array}{l}\text { Chronic } \\
\text { Insomnia } \\
\text { Disorder }\end{array}$} & \multirow[t]{2}{*}{$\begin{array}{l}\text { Healthy } \\
\text { Controls }\end{array}$} & \multirow[t]{2}{*}{ Statistic } & \multirow[t]{2}{*}{$P$ value } & \multicolumn{3}{|c|}{$\begin{array}{l}P \text { value in Multiple } \\
\text { Comparisons }\end{array}$} \\
\hline & & & & & & $\begin{array}{l}\text { ICD vs } \\
\text { CID }\end{array}$ & $\begin{array}{l}\text { ICD vs } \\
\text { CON }\end{array}$ & $\begin{array}{l}\text { CID vs } \\
\text { CON }\end{array}$ \\
\hline Number of cases & 42 & 63 & 42 & & & & & \\
\hline Male/female & $17 / 25$ & $20 / 43$ & $22 / 20$ & $C^{2}=2.7$ & 0.3 & & & \\
\hline Age, years & $39.6 \pm 12.1$ & $41.3 \pm 12.3$ & $4|.4 \pm| 4.1$ & $\mathrm{~F}=0.3$ & 0.7 & & & \\
\hline Education, years & $12.0(6.0,16.0)$ & $12.0(9.0,16.0)$ & $15.0(9.0,16.0)$ & $\mathrm{H}=3.1$ & 0.2 & & & \\
\hline BMI & $21.7 \pm 3.1$ & $21.7 \pm 2.6$ & - & $t=0.02$ & 0.98 & & & \\
\hline HAMD-I7 & $19.0(13.8,22.0)^{* \#}$ & $10.0(7.0,13.0)^{*}$ & $2.0(1.0,4.3)$ & $\mathrm{H}=104.0$ & $<0.001$ & $<0.001$ & $<0.001$ & $<0.001$ \\
\hline PSQI & $17.0(15.0,19.0)^{*}$ & $15.0(\mid 3.0,17.0)^{*}$ & $2.0(0.0,3.3)$ & $\mathrm{H}=92.2$ & $<0.001$ & 0.13 & $<0.001$ & $<0.001$ \\
\hline
\end{tabular}

Notes: Normally distributed variables are shown as mean \pm SD; non-normally distributed variables are shown as $P 50$ (P25, P75). ${ }^{*} P<0.05$ vs healthy controls. ${ }^{\#} P<0.01$ vs chronic insomnia disorder.

Abbreviations: BMI, body mass index; CID, chronic insomnia disorder; CON, control; HAMD-I7, Hamilton Depression Rating Scale (I7 items); ICD, insomnia comorbid with depression; PSQI, Pittsburgh Sleep Quality Index.

\section{Serum Cytokine Levels}

There were significant intergroup differences in serum levels of IL-1RA ( $\mathrm{H}=9.85, P=0.007), \mathrm{IL}-4 \quad(\mathrm{H}=15.85$, $P<0.001), \mathrm{IL}-5 \quad(\mathrm{H}=14.77, P=0.001), \mathrm{IL}-10 \quad(\mathrm{H}=31.58$, $P<0.001), \mathrm{IL}-13(\mathrm{H}=14.37, P=0.001)$, IL-28A $(\mathrm{H}=17.55$, $P<0.001)$, and GM-CSF $(\mathrm{H}=18.37, \quad P<0.001)$. The results of multiple comparisons indicated that the ICD group had significantly lower levels of IL-1RA
$(P=0.01), \quad$ IL-4 $\quad(P=0.03), \quad$ IL-5 $\quad(P=0.02), \quad$ IL-10 $(P<0.001)$, and IL-28A $(P=0.02)$ than control subjects, and lower levels of IL-1RA $(P=0.02)$, IL-5 $(P=0.002)$, IL-10 $\quad(P<0.001), \quad$ IL-13 $\quad(P=0.001), \quad$ and GM-CSF $(P=0.02)$ than the CID group. Moreover, the CID group had elevated IL-13 and GM-CSF $(P=0.03$; $P<0.001)$ and reduced IL-4 and IL-28A $(P=0.001$; $P<0.001$ ) levels relative to controls (Table 3).

Table 2 Cognitive Performance of the Study Subjects

\begin{tabular}{|c|c|c|c|c|c|c|c|c|c|}
\hline \multirow{2}{*}{\multicolumn{2}{|c|}{ Terms }} & \multirow[t]{2}{*}{ ICD } & \multirow[t]{2}{*}{ CID } & \multirow[t]{2}{*}{ CON } & \multirow[t]{2}{*}{$\mathbf{H}$} & \multirow[t]{2}{*}{$P$ value } & \multicolumn{3}{|c|}{$\begin{array}{l}P \text { value in Multiple } \\
\text { Comparisons }\end{array}$} \\
\hline & & & & & & & $\begin{array}{l}\text { ICD } \\
\text { vs } \\
\text { CID }\end{array}$ & $\begin{array}{l}\text { ICD vs } \\
\text { CON }\end{array}$ & $\begin{array}{l}\text { CID vs } \\
\text { CON }\end{array}$ \\
\hline \multicolumn{2}{|c|}{ MoCA-C (scores) } & $26.5(25.0,28.0)$ & $27.0(25.0,29.0)$ & $27.0(26.0,28.0)$ & 1.9 & 0.4 & & & \\
\hline \multirow{5}{*}{$\begin{array}{l}\text { Nine- } \\
\text { box } \\
\text { maze }\end{array}$} & ORM (errors) & $0.0(0.0,1.0)$ & $0.0(0.0,1.0)$ & $0.0(0.0,1.0)$ & 0.8 & 0.7 & & & \\
\hline & SRM (errors) & $1.0(0.0,2.0)^{* \#}$ & $0.0(0.0,2.0)$ & I.0 (0.0, I.0) & 9.4 & 0.01 & 0.01 & 0.03 & 1.00 \\
\hline & OWM (errors) & $0.0(0.0,1.0)$ & $0.0(0.0,0.0)$ & $0.0(0.0,1.0)$ & 2.4 & 0.3 & & & \\
\hline & SWM (errors) & $4.0(3.0,8.3)^{*}$ & $4.0(2.0,5.0)^{*}$ & $1.0(1.0,3.0)$ & 37.6 & $<0.001$ & 0.56 & $<0.001$ & $<0.001$ \\
\hline & ORcM (errors) & $0.0(0.0,0.0)$ & $0.0(0.0,0.0)^{*}$ & $0.0(0.0,0.0)$ & 8.8 & 0.01 & 0.25 & 0.95 & 0.01 \\
\hline
\end{tabular}

Notes: Normally distributed variables are shown as mean \pm SD; non-normally distributed variables are shown as $\mathrm{P} 50$ (P25, $\mathrm{P} 75)$. ${ }^{*} P<0.05$ vs healthy controls. ${ }^{*} P<0.01$ vs chronic insomnia disorder.

Abbreviations: CID, chronic insomnia disorder; CON, control; ICD, insomnia comorbid with depression; MoCA-C, Chinese-Beijing Version of Montreal Cognitive Assessment Scale; ORcM, object recognition memory; ORM, object reference memory; OWM, object working memory; SRM, spatial reference memory; SWM, spatial working memory. 


\section{Correlations Among Serum Cytokine Levels, Depression Severity, Sleep Quality, and Cognitive Function}

In the ICD group, partial correlation analysis between serum cytokine levels and cognitive performance showed that IL-4 level was positively correlated with the number of errors in OWM ( $r=0.39, P=0.04)$ while IL-5 level was correlated with number of errors in OWM $(r=0.39$, $P=0.04)$ and ORcM $(r=0.58, P<0.01)$, IL-10 level was also positively correlated with the number of errors in 3 tasks - ie, ORM ( $r=0.40, P=0.03), \operatorname{SRM}(r=0.57, P<0.01)$, and ORcM $(r=0.47, P=0.01)$, and IL-13 $(r=0.51, P<0.01)$ and TGF- $\beta 1(r=0.51, P<0.01)$ after controlling for sex, age, education, BMI, and PSQI and HAMD-17 scores (Table 4). In the CID group, the identical partial correlation analysis showed that serum concentration of IL-1RA was positively correlated with ORM errors $(r=0.34$, $P=0.01)$, whereas IL-5 $(r=0.37, P<0.001)$, IL-10 $(r=0.28$, $P=0.03)$, and IL-13 $(r=0.40, P<0.001)$ levels were positively correlated with OWM errors.

\section{Discussion}

\section{ICD Patients Had Decreased Serum} Levels of Anti-Inflammatory Cytokines and GM-CSF

Pro-inflammatory and anti-inflammatory mediators as the two classed of molecules so-exist and cause effects jointly in actual pathological process. ${ }^{44}$ Immune changes in depression and insomnia resulting from the activation of proinflammatory cytokines. ${ }^{45-47}$ However, other cytokines including anti-inflammatory ILs, TGF, GM-CSF, IFN, and RANTES have received less attention. In the current study, we tried to examine serum cytokine profiles (focusing on the abovementioned previously overlooked cytokines) in ICD to evaluate their change and its relationship with cognitive impairments. Our findings showed that patients with ICD had lower serum levels of anti-inflammatory cytokines and GM-CSF than patients with CID and control subjects. The results indicate that ICD as a comorbidity disease may present lower serum levels of antiinflammatory cytokines and GM-CSF. Considering an imbalance between pro- and anti-inflammatory cytokines triggers the onset of depression ${ }^{48}$; we proposed that such an imbalance - caused by decreased anti-inflammatory and increased proinflammatory cytokine levels - exists in ICD. The reason may be the activation of serine kinases by ATP on inflammatory response. ${ }^{49}$ By interacting with the purinergic receptor $\mathrm{P} 2 \mathrm{X} 7$, eATP triggers the secretion of proinflammatory cytokines. Additionally, it may inhibit the inflammatory response through an interaction with the receptor $\mathrm{P} 2 \mathrm{Y}$ and its downstream cAMP-PKA pathway. ${ }^{50,51}$

\section{ICD Patients Have Cognitive Impairment Compared with CID Patients}

Cognitive dysfunction is a frequent complaint of patients with depression or insomnia. ${ }^{8}$ Cognitive impairment associated with depression includes reduced daytime functioning and deficits in attention, memory, psychomotor processing, and problem solving. ${ }^{9}$ Individuals who have insomnia show poor performance in objective measures of perceptual function, manipulation and retention capacity in working memory, complex attention, alertness, episodic memory, and problem solving relative to control subjects. ${ }^{52}$ Our preliminary study showed that ICD group had more errors in all five measures of memory (SRM, SWM, ORM, and ORcM) compared to controls. ${ }^{42}$ However, in the CID group, there had some difference between our previous researches about spatial memory evaluation. In the present study, patients with ICD only showed impairment in SRM and SWM. Consistent with Chen's study, we found here that patients with CID had more errors in SWM and ORcM than control subjects. ${ }^{43}$ The results clearly implying that the nature of cognitive impairment differs between ICD and CID, which may help us distinguish ICD from CID. This discrepancy may be attributable to factors such as the illness duration, the existence of heterogeneity in the current study, sample size, and differences in the severity of insomnia. ${ }^{53-56}$ Further studies are needed to reach a definitive conclusion.

\section{Relationship Between Serum Cytokine Levels and Sleep Quality and Cognitive Function}

Inflammation in acute and chronic diseases has been shown to contribute to poor sleep quality. ${ }^{57,58}$ The sympathetic nervous system (SNS) and hypothalamic-pituitaryadrenal (HPA) axis are the main systems linking sleep and immune function. ${ }^{59}$ The increased excitability of the SNS leading to overexpression of inflammation-related genes, overproduction of inflammatory cytokines, and increased systemic inflammation. ${ }^{60}$ Different levels of antiinflammatory cytokines such as IL-10 and IL-13 have 


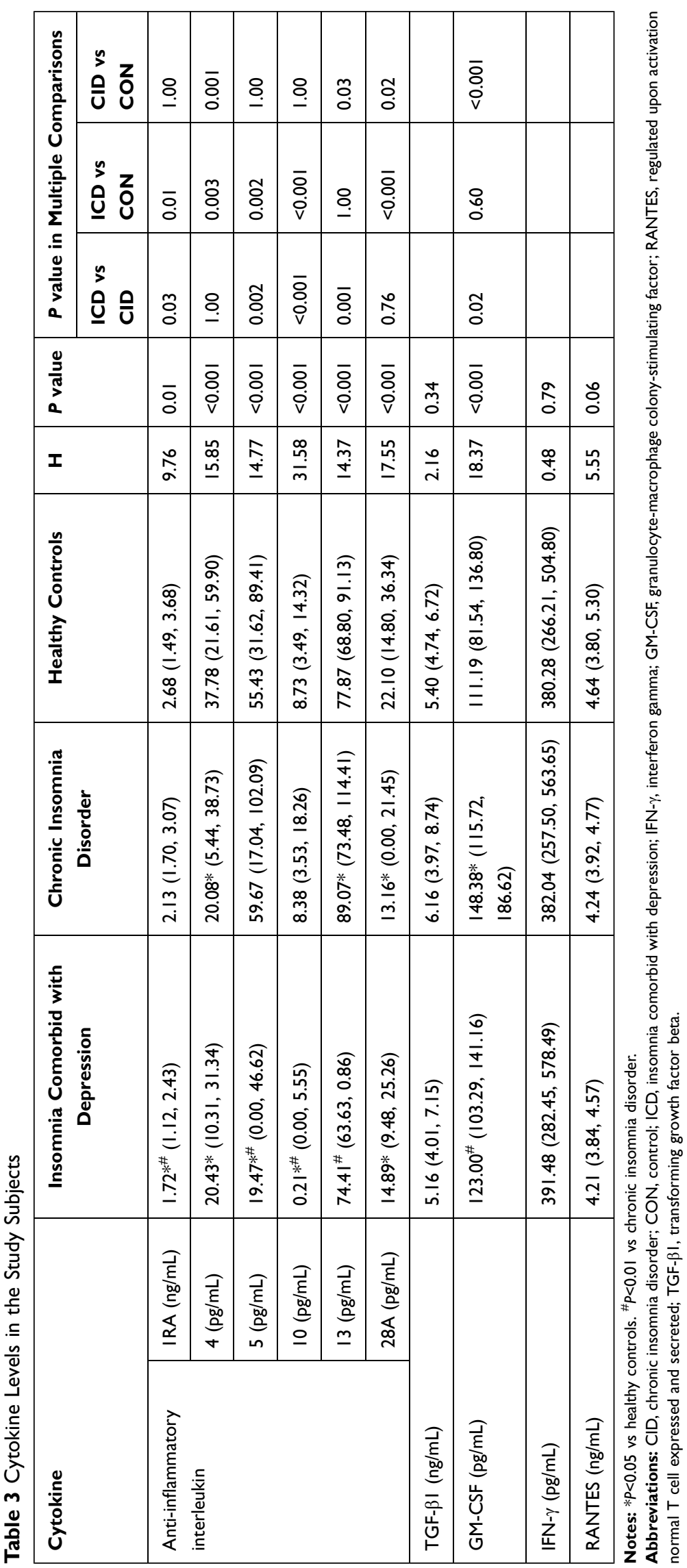


Table 4 Partial Correlations Among Serum Cytokines and HAMD-I7, PSQI, and MoCA Scores and Spatial Memory in Patients with Insomnia Comorbid with Depression and Chronic Insomnia Disorder

\begin{tabular}{|c|c|c|c|c|c|c|c|c|c|c|}
\hline \multirow[t]{2}{*}{ Disorder } & \multirow{2}{*}{\multicolumn{2}{|c|}{ Cytokine }} & \multirow{2}{*}{$\begin{array}{l}\text { HAMD- } \\
\qquad 17^{\mathrm{a}}\end{array}$} & \multirow{2}{*}{$\underset{\text { PS }}{\text { PSQ }}$} & \multirow{2}{*}{$\begin{array}{c}\text { MoCA- } \\
\text { C }^{c}\end{array}$} & \multicolumn{5}{|c|}{ Spatial Memories $^{c}$} \\
\hline & & & & & & ORM & SRM & OWM & SWM & ORcM \\
\hline \multirow[t]{10}{*}{ ICD } & Anti-inflammatory & IRA $(\mathrm{ng} / \mathrm{mL})$ & -0.06 & 0.04 & 0.11 & -0.09 & -0.20 & -0.02 & -0.12 & 0.09 \\
\hline & interleukin & $4(\mathrm{pg} / \mathrm{mL})$ & -0.13 & 0.30 & 0.24 & 0.04 & 0.08 & $0.39 *$ & -0.07 & 0.29 \\
\hline & & $5(\mathrm{pg} / \mathrm{mL})$ & -0.05 & 0.09 & 0.01 & 0.20 & 0.35 & $0.39 *$ & -0.20 & $0.58^{\#}$ \\
\hline & & $10(\mathrm{pg} / \mathrm{mL})$ & -0.19 & 0.10 & -0.17 & $0.40 *$ & $0.57^{\#}$ & 0.21 & -0.25 & $0.47^{*}$ \\
\hline & & $13(\mathrm{pg} / \mathrm{mL})$ & 0.09 & -0.16 & $0.5 I^{\#}$ & -0.30 & -0.15 & -0.18 & -0.13 & -0.01 \\
\hline & & $28 \mathrm{~A}(\mathrm{pg} / \mathrm{mL})$ & 0.01 & -0.09 & -0.15 & 0.17 & 0.14 & 0.33 & 0.00 & 0.30 \\
\hline & \multicolumn{2}{|l|}{ TGF- $\beta$ I (ng/mL) } & 0.12 & -0.12 & $0.5 I^{\#}$ & -0.26 & -0.16 & -0.29 & 0.06 & 0.10 \\
\hline & \multicolumn{2}{|l|}{ GM-CSF (pg/mL) } & 0.10 & -0.10 & 0.24 & 0.07 & 0.11 & -0.24 & 0.16 & 0.17 \\
\hline & \multicolumn{2}{|l|}{$\mathrm{IFN}-\gamma(\mathrm{pg} / \mathrm{mL})$} & -0.21 & -0.13 & 0.16 & -0.32 & -0.20 & -0.24 & 0.15 & -0.04 \\
\hline & \multicolumn{2}{|l|}{ RANTES (ng/mL) } & 0.21 & 0.24 & 0.27 & 0.23 & 0.20 & 0.15 & -0.35 & 0.10 \\
\hline \multirow[t]{10}{*}{ CID } & Anti-inflammatory & IRA (ng/mL) & 0.02 & 0.21 & -0.05 & $0.34^{*}$ & 0.11 & 0.15 & -0.12 & -0.03 \\
\hline & interleukin & $4(\mathrm{pg} / \mathrm{mL})$ & -0.15 & -0.04 & 0.01 & -0.10 & -0.06 & 0.15 & -0.11 & -0.12 \\
\hline & & $5(\mathrm{pg} / \mathrm{mL})$ & -0.14 & 0.08 & 0.10 & -0.01 & -0.05 & $0.37^{\#}$ & -0.19 & 0.07 \\
\hline & & $10(\mathrm{pg} / \mathrm{mL})$ & -0.18 & 0.13 & 0.11 & -0.10 & -0.06 & $0.28 *$ & -0.20 & 0.10 \\
\hline & & $13(\mathrm{pg} / \mathrm{mL})$ & -0.02 & 0.05 & -0.25 & -0.08 & -0.01 & $0.40^{\#}$ & 0.03 & 0.04 \\
\hline & & $28 \mathrm{~A}(\mathrm{pg} / \mathrm{mL})$ & 0.04 & -0.20 & -0.05 & -0.11 & -0.20 & 0.00 & 0.18 & -0.02 \\
\hline & \multicolumn{2}{|l|}{ TGF- $\beta$ I (ng/mL) } & -0.01 & 0.04 & -0.02 & -0.07 & 0.15 & 0.21 & -0.05 & 0.10 \\
\hline & \multicolumn{2}{|l|}{ GM-CSF (pg/mL) } & -0.16 & 0.08 & -0.15 & -0.07 & 0.14 & 0.23 & 0.04 & 0.00 \\
\hline & \multicolumn{2}{|l|}{ IFN- $\gamma(\mathrm{pg} / \mathrm{mL})$} & 0.01 & 0.03 & -0.16 & -0.10 & 0.10 & 0.23 & -0.10 & -0.03 \\
\hline & \multicolumn{2}{|l|}{ RANTES (ng/mL) } & 0.11 & 0.15 & -0.05 & -0.10 & -0.19 & -0.03 & -0.18 & 0.02 \\
\hline
\end{tabular}

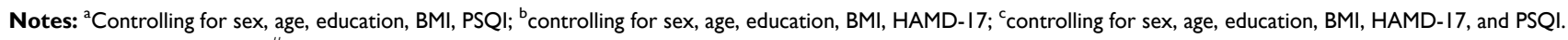
$* P<0.05$ vs healthy controls. ${ }^{\#} P<0.01$ vs chronic insomnia disorder.

Abbreviations: CID, chronic insomnia disorder; GM-CSF, granulocyte-macrophage colony-stimulating factor; HAMD-I7, Hamilton Depression Rating Scale (I7 items); ICD, insomnia comorbid with depression; IFN- $\gamma$, interferon gamma; MoCA-C, Chinese-Beijing Version of Montreal Cognitive Assessment Scale; ORcM, object recognition memory; ORM, object reference memory; OWM, object working memory; PSQI, Pittsburgh Sleep Quality Index; RANTES, regulated upon activation normal T cell expressed and secreted; SRM, spatial reference memory; SWM, spatial working memory; TGF- $\beta$ I, transforming growth factor beta I.

been observed cause the reduction at non-rapid eye movement (NREM) sleep in both humans and animal models. $^{61-63}$ In the present study, we did not find a significant correlation between anti-inflammatory cytokine levels and subjective sleep quality as indicated by PSQI score in patients with ICD. Our results were consistent with Petrov's clinical research about subjective sleep quality and inflammation in healthy subjects. ${ }^{64}$ Additionally, individuals with insomnia tend to overestimate time to fall asleep time in comparison to PSG measures. ${ }^{65}$ Thus, subjective sleep measures for insomnia are associated with unsatisfactory diagnostic accuracy. ${ }^{66}$ Considering the relationship between serum cytokines and subjective sleep quality still controversial and our results cannot conclusively prove that anti-inflammatory cytokines correlated with subjective sleep quality, ${ }^{67,68}$ we consider the possible reason may be attributable to the existence of comorbidities, various degree of insomnia severity, lack of objective tools for evaluating sleep quality (eg, polysomnography), and classification of different types of insomnia. ${ }^{69-71}$

Cytokines have been implicated in a variety of cognitive processes such as learning, memory, and executive function. ${ }^{72,73}$ A recent study about insomnia patients indicates the role of brain-derived neurotrophic factor (BDNF) gene polymorphism in cognitive impairment. ${ }^{74}$ Systemic changes in cytokine levels have been linked to several types of cognitive disorder including Alzheimer disease and mild cognitive impairment. ${ }^{75}$ Proinflammatory cytokines modulate hippocampal function by stimulating the production of oxygen free radicals, leading to cognitive impairment. $^{76}$ Meanwhile, anti-inflammatory cytokines 
such as IL-4, IL-10, and IL-13 can inhibit the activation of microglia to suppress inflammation. ${ }^{76}$ Thus, when the levels of anti-inflammatory cytokines are reduced, the deleterious effects of proinflammatory cytokines on cognitive function cannot be mitigated. ${ }^{77,78}$ In the present work, we found that elevated levels of IL-4, IL-5, IL-10, IL-13, and TGF- $\beta 1$ had positive effects on cognitive function (as evaluated by MoCA and a spatial memory task) in ICD patients. Thus, anti-inflammatory cytokines may actively participate in normal memory functions and conversely, their reduction could lead to cognitive deficits in pathologic states such as ICD. ${ }^{79}$ Notably, our results also demonstrated that the levels of another set of antiinflammatory cytokines (IL-1RA, IL-5, IL-10, and IL-13) were positively correlated with object memory (ORM or OWM) in CID patients, which was similar with the cognitive domains associated with anti-inflammatory cytokines in ICD. In view of both OWM and ORM belong to object memory, our finding suggested that antiinflammatory cytokines are positively correlated with object memory and the inhibition of these cytokines may be a potential treatment strategy to relieve cognitive dysfunction. ${ }^{80}$ The possible mechanisms may be due to the presence of high levels of pro-inflammatory cytokines, the production or action of anti-inflammatory cytokines are suppressed, abolishing the beneficial effects of antiinflammatory cytokines on learning and memory. ${ }^{81}$

\section{Limitations}

The current study had several limitations. First, given this was a cross-sectional study, we could not draw causal inference and the inflammatory factors were only measured at initial presentation rather than being tracked during the treatment. Therefore, the relationship between ICD and anti-inflammatory cytokines with cognitive impairments could not be determined casually. For the same reason, the analysis of cytokines that we do not account for the improvement in depression and lack of a control group only with depression without insomnia, which would be another limitation. Second, there is still controversy about whether insomnia is a specific disease or a symptom comorbid with other diseases. The latest diagnostic of ICSD-3 has stressed the existence of insomnia as a specific disease. ${ }^{12}$ However, for patients comorbid with depression and insomnia, it is hard to distinguish the causal relationship between depression and insomnia and whether insomnia is a specific disease or a depressive symptom. Third, a small sample size, little data on baseline characteristics and a lack of objective instrument to evaluate sleep quality, which may affect the results. Further prospective studies are therefore required to overcome these limitations and further verify the generalization of the results in this study.

\section{Conclusion}

Individuals with ICD showed decreased levels of antiinflammatory ILs, suggesting immune regulation as a possible mechanism underlying this disorder. Additionally, ICD patients had cognitive impairmentspecifically, related to spatial memory, and the cognitive deficits were associated with altered serum cytokine levels. Our results suggest that ICD is a distinct condition that can be distinguished from CID based on serum cytokine profile and specific types of cognitive dysfunction.

\section{Abbreviations}

ATP, adenosine triphosphate; BDNF, brain-derived neurotrophic factor; BMI, body mass index; CID, chronic insomnia disorder; DSM-5, Diagnostic and Statistical Manual of Mental Disorders, Fifth Edition; eATP, extracellular ATP; GM-CSF, granulocyte-macrophage colonystimulating factor; HAMD-17, 17-Item Hamilton Depression Rating Scale; HPA, hypothalamic-pituitaryadrenal; ICD, insomnia comorbid with depression; ICSD-3, International Classification of Sleep Disorders, Third Edition; IFN, interferon; IL, interleukin; Mini 5.0, Mini International Neuropsychiatry Interview 5.0; MoCA, Montreal Cognitive Assessment; MoCA-C, ChineseBeijing Version of Montreal Cognitive Assessment; NBMT, Nine-Box Maze Test; NREM, non-rapid eye movement; ORcM, object recognition memory; ORM, object reference memory; OWM, object working memory; PSQI, Pittsburgh Sleep Quality Index; RANTES, regulated upon activation, normal $\mathrm{T}$ cell expressed and secreted; SNS, sympathetic nervous system; SRM, spatial reference memory; SWM, spatial working memory; TGF$\beta 1$, transforming growth factor beta 1 ; TNF, tumor necrosis factor.

\section{Acknowledgments}

We would like to thank all patients who participated in our study. This work was financially supported by the National Natural Science Foundation of China (grant no. 81671316) and Natural Science Foundation of Anhui Medical University (grant nos. 2018xkj029 and 2018xkj066). These funding sources had no role in the study design, 
analysis, data interpretation, or decision to submit the article for publication.

\section{Disclosure}

The authors declare no conflicts of interest in this work.

\section{References}

1. Murphy MJ, Peterson MJ. Sleep disturbances in depression. Sleep Med Clin. 2015;10(1):17-23. doi:10.1016/j.jsmc.2014.11.009

2. Hertenstein E, Feige B, Gmeiner T, et al. Insomnia as a predictor of mental disorders: a systematic review and meta-analysis. Sleep Med Rev. 2019;43:96-105. doi:10.1016/j.smrv.2018.10.006

3. Pigeon WR, Bishop TM, Krueger KM. Insomnia as a precipitating factor in new onset mental illness: a systematic review of recent findings. Curr Psychiatry Rep. 2017;19(8):44. doi:10.1007/s11920017-0802-x

4. Chen PJ, Huang CLC, Weng SF, et al. Relapse insomnia increases greater risk of anxiety and depression: evidence from a population-based 4-year Cohort Study. Sleep Med. 2017;38:122-129. doi:10.1016/j.sleep.2017.07.016

5. Koyama F, Yoda T, Hirao T. Insomnia and depression: Japanese hospital workers questionnaire survey. Open Med. 2017;12 (1):391-398. doi:10.1515/med-2017-0056

6. Fang H, Tu S, Sheng J, Shao A. Depression in sleep disturbance: a review on a bidirectional relationship, mechanisms and treatment. J Cell Mol Med. 2019;23(4):2324-2332. doi:10.1111/jcmm.14170

7. Sivertsen B, Salo P, Mykletun A, et al. The bidirectional association between depression and insomnia: the HUNT Study. Psychosom Med. 2012;74(7):758-765. doi:10.1097/PSY.0b013e3182648619

8. Pang R, Guo R, Wu X, et al. Altered regional homogeneity in chronic insomnia disorder with or without cognitive impairment. $\mathrm{Am}$ J Neuroradiol. 2018;39(4):742-747. doi:10.3174/ajnr.A5587

9. Culpepper L, Lam RW, McIntyre RS. Cognitive impairment in patients with depression: awareness, assessment, and management. $J \quad$ Clin Psychiatry. 2017;78(9):1383-1394. doi:10.4088/JCP. tk16043ah5c

10. Knight MJ, Baune BT. Cognitive dysfunction in major depressive disorder. Curr Opin Psychiatry. 2018;31(1):26-31. doi:10.1097/ YCO.0000000000000378

11. American Psychiatric Association. Diagnostic and Statistical Manual of Mental Disorders. 5th ed. Washington, DC: American Psychiatric Association; 2013.

12. Morin CM, Drake CL, Harvey AG, et al. Insomnia disorder. Nat Rev Dis Primers. 2015;1(1):1-18. doi:10.1038/nrdp.2015.26

13. Tsoupras A, Lordan R, Zabetakis I. Inflammation, not cholesterol, is a cause of chronic disease. Nutrients. 2018;10(5):604. doi:10.3390/ nu10050604

14. Wolf D, Ley K. Immunity and inflammation in atherosclerosis. Circ Res. 2019;124(2):315-327. doi:10.1161/CIRCRESAHA.118.313591

15. Hodes GE, Pfau ML, Leboeuf M, et al. Individual differences in the peripheral immune system promote resilience versus susceptibility to social stress. Proc Natl Acad Sci U S A. 2014;111(45):16136-16141. doi:10.1073/pnas.1415191111

16. Lamers F, Milaneschi Y, Smit JH, Schoevers RA, Wittenberg G, Penninx BWJH. Longitudinal association between depression and inflammatory markers: results from the Netherlands study of depression and anxiety. Biol Psychiatry. 2019;85(10):829-837. doi:10.1016/ j.biopsych.2018.12.020

17. Martín-Hernández D, Tendilla-Beltrán H, Madrigal JLM, García-Bueno B, Leza JC, Caso JR. Chronic mild stress alters kynurenine pathways changing the glutamate neurotransmission in frontal cortex of rats. Mol Neurobiol. 2019;56(1):490-501. doi:10.1007/ s12035-018-1096-7
18. Strawbridge R, Arnone D, Danese A, Papadopoulos A, Herane Vives A, Cleare AJ. Inflammation and clinical response to treatment in depression: a meta-analysis. Eur Neuropsychopharmacol. 2015;25 (10):1532-1543. doi:10.1016/j.euroneuro.2015.06.007

19. Thomas J, Khanam R, Vohora D. Activation of indoleamine 2, 3-dioxygenase pathway by olanzapine augments antidepressant effects of venlafaxine in mice. Psychiatry Res. 2017;258:444-448. doi:10.1016/j.psychres.2017.08.083

20. Köhler-Forsberg O, N. Lydholm C, Hjorthøj C, Nordentoft M, Mors O, Benros ME. Efficacy of anti-inflammatory treatment on major depressive disorder or depressive symptoms: meta-analysis of clinical trials. Acta Psychiatr Scand. 2019;139(5):404-419. doi:10.1111/acps.13016

21. Zou W, Feng R, Yang Y. Changes in the serum levels of inflammatory cytokines in antidepressant drug-naïve patients with major depression. PLoS One. 2019;13:1-10. doi:10.1371/journal.pone.0197267

22. Misiak B, Beszłej JA, Kotowicz K, et al. Cytokine alterations and cognitive impairment in major depressive disorder: from putative mechanisms to novel treatment targets. Prog Neuropsychopharmacology Biol Psychiatry. 2018;80:177-188. doi:10.1016/j.pnpbp.2017.04.021

23. Besedovsky L, Lange T, Haack M. The sleep-immune crosstalk in health and disease. Physiol Rev. 2019;99(3):1325-1380. doi:10.1152/ physrev.00010.2018

24. Irwin MR, Olmstead R, Carrillo $C$, et al. Cognitive behavioral therapy vs. Tai Chi for late life insomnia and inflammatory risk: a randomized controlled comparative efficacy trial. Sleep. 2014;37 (9):1543-1552. doi:10.5665/sleep.4008

25. Oppenheim JJ. The future of the cytokine discipline. Cold Spring Harb Perspect Biol. 2018;10:1-8. doi:10.1101/cshperspect.a028498

26. Borish LC, Steinke JW. 2. Cytokines and chemokines. J Allergy Clin Immunol. 2003;111(2):460-475. doi:10.1067/mai.2003.108

27. Li Y, Wei $\mathrm{C}, \mathrm{Xu} \mathrm{H}$, et al. The immunoregulation of Th17 in host against intracellular bacterial infection. Mediators Inflamm. 2018;2018:6587296. doi:10.1155/2018/6587296

28. Spolski R, Leonard WJ. Interleukin-21: basic biology and implications for cancer and autoimmunity. Апnи Rev Immunol. 2008;26 (1):57-79. doi:10.1146/annurev.immunol.26.021607.090316

29. Witte K, Witte E, Sabat R, Wolk K. IL-28A, IL-28B, and IL-29: promising cytokines with type I interferon-like properties. Cytokine Growth Factor Rev. 2010;21(4):237-251. doi:10.1016/j.cytogfr.2010.04.002

30. Himmerich H, Patsalos O, Lichtblau N, Ibrahim MAA, Dalton B. Cytokine research in depression: principles, challenges, and open questions. Front Psychiatry. 2019;10:30. doi:10.3389/ fpsyt.2019.00030

31. Petralia MC, Mazzon E, Fagone P, et al. The cytokine network in the pathogenesis of major depressive disorder. Close to translation? Autoimmun Rev. 2020;19(5):102504. doi:10.1016/j.autrev.2020.102504

32. Schmidt FM, Lichtblau N, Minkwitz J, et al. Cytokine levels in depressed and non-depressed subjects, and masking effects of obesity. J Psychiatr Res. 2014;55:29-34. doi:10.1016/j.jpsychires.2014.04.021

33. Fourrier C, Singhal G, Baune BT. Neuroinflammation and cognition across psychiatric conditions. CNS Spectr. 2019;24(1):4-15. doi:10.1017/S1092852918001499

34. Carpenter JS, Andrykowski MA. Psychometric evaluation of the Pittsburgh sleep quality index. J Psychosom Res. 1998;45(1):5-13. doi:10.1016/S0022-3999(97)00298-5

35. Mollayeva T, Thurairajah P, Burton K, Mollayeva S, Shapiro CM, Colantonio A. The Pittsburgh sleep quality index as a screening tool for sleep dysfunction in clinical and non-clinical samples: a systematic review and meta-analysis. Sleep Med Rev. 2016;25:52-73. doi:10.1016/j.smrv.2015.01.009

36. Tsai PS, Wang SY, Wang MY, et al. Psychometric evaluation of the Chinese version of the Pittsburgh Sleep Quality Index (CPSQI) in primary insomnia and control subjects. Qual Life Res. 2005;14 (8):1943-1952. doi:10.1007/s11136-005-4346-x 
37. Hamilton M. A rating scale for depression. $J$ Neurol Neurosurg Psychiatry. 1960;23(1):56-62. doi:10.1136/jnnp.23.1.56

38. Si TM, Shu L, Dang WM, et al. Evaluation of the reliability and validity of Chinese version of mini-international neuropsychiatric interview in patients with mental disorders. Chin Ment Health J. 2009;023(007):493-497, 503. doi:10.3969/j.issn.10006729.2009.07.011

39. Chen X, Zhang R, Xiao Y, Dong J, Niu X, Kong W. Reliability and validity of the Beijing version of the Montreal cognitive assessment in the evaluation of cognitive function of adult patients with OSAHS PLoS One. 2015;10:1-12. doi:10.1371/journal.pone.0132361

40. Hsu JL, Fan YC, Huang YL, et al. Improved predictive ability of the Montreal cognitive assessment for diagnosing dementia in a community-based study. Alzheimers Res Ther. 2015;7(1):69. doi:10.1186/s13195-015-0156-8

41. Abrahams S, Pickering A, Polkey CE, Morris RG. Spatial memory deficits in patients with unilateral damage to the right hippocampal formation. Neuropsychologia. 1997;35(1):11-24. doi:10.1016/S00283932(96)00051-6

42. Porter VR, Buxton WG, Avidan AY. Sleep, cognition and dementia. Curr Psychiatry Rep. 2015;17(12):97. doi:10.1007/s11920-015-0631-8

43. Chen GH, Xia L, Wang F, Li XW, Jiao CA. Patients with chronic insomnia have selective impairments in memory that are modulated by cortisol. Psychophysiology. 2016;53(10):1567-1576. doi:10.1111/ psyp. 12700

44. Mao R, Zhang C, Chen J, et al. Different levels of pro- and anti-inflammatory cytokines in patients with unipolar and bipolar depression. $J$ Affect Disord. 2018;237:65-72. doi:10.1016/j. jad.2018.04.115

45. Dolsen MR, Soehner AM, Harvey AG. Proinflammatory cytokines, mood, and sleep in interepisode bipolar disorder and insomnia: a pilot study with implications for psychosocial interventions. Psychosomatic Med. 2018;80(1):87-94. doi:10.1097/PSY.0000000000000529

46. Felger JC, Lotrich FE. Inflammatory cytokines in depression: neurobiological mechanisms and therapeutic implications. Neuroscience. 2013;246:199-229. doi:10.1016/j.neuroscience.2013.04.060

47. Howren MB, Lamkin DM, Suls J. Associations of depression with c-reactive protein, IL-1, and IL-6: a meta-analysis. Psychosom Med. 2009;71(2):171-186. doi:10.1097/PSY.0b013e3181907c1b

48. Kim YK, Na KS, Myint AM, Leonard BE. The role of pro-inflammatory cytokines in neuroinflammation, neurogenesis and the neuroendocrine system in major depression. Prog Neuropsychopharmacol Biol Psychiatry. 2016;64:277-284. doi:10.1016/j.pnpbp.2015.06.008

49. Lee JH, Zhang Y, Zhao Z, et al. Intracellular ATP in balance of proand anti-inflammatory cytokines in adipose tissue with and without tissue expansion. Int $J$ Obes (Lond). 2017;41(4):645-651. doi:10.1038/ijo.2017.3

50. Ham J, Rees DA. The adenosine $a 2 b$ receptor: its role in inflammation. Endocr Metab Immune Disord Drug Targets. 2008;8 (4):244-254. doi:10.2174/187153008786848303

51. Vitiello L, Gorini S, Rosano G, la Sala A. Immunoregulation through extracellular nucleotides. Blood. 2012;120(3):511-518. doi:10.1182 blood-2012-01-406496

52. Wardle-Pinkston S, Slavish DC, Taylor DJ. Insomnia and cognitive performance: a systematic review and meta-analysis. Sleep Med Rev 2019;48:101205. doi:10.1016/j.smrv.2019.07.008

53. Ren CY, Rao JX, Zhang XX, Zhang M, Xia L, Chen GH. Changed signals of blood adenosine and cytokines are associated with parameters of sleep and/or cognition in the patients with chronic insomnia disorder. Sleep Med. 2021;81:42-51. doi:10.1016/j. sleep.2021.02.005

54. Shekleton JA, Flynn-Evans EE, Miller B, et al. Neurobehavioral performance impairment in insomnia: relationships with self-reported sleep and daytime functioning. Sleep. 2014;37 (1):107-116. doi: $10.5665 /$ sleep. 3318
55. Li Y, Liu L, Wang E, et al. Abnormal neural network of primary insomnia: evidence from spatial working memory task fMRI. Eur Neurol. 2016;75(1-2):48-57. doi:10.1159/000443372

56. Fan TT, Chen WH, Shi L, et al. Objective sleep duration is associated with cognitive deficits in primary insomnia: BDNF may play a role. Sleep. 2019;42(1):10.1093/sleep/zsy192. doi:10.1093/sleep/ zsy 192

57. Roehrs T, Diederichs C, Gillis M, et al. Nocturnal sleep, daytime sleepiness and fatigue in fibromyalgia patients compared to rheumatoid arthritis patients and healthy controls: a Preliminary Study. Sleep Med. 2013;14(1):109-115. doi:10.1016/j.sleep.2012.09.020

58. Sharpley AL, Cooper CM, Williams C, Godlewska BR, Cowen PJ. Effects of typhoid vaccine on inflammation and sleep in healthy participants: a Double-Blind, Placebo-Controlled, Crossover Study. Psychopharmacology (Berl). 2016;233(18):3429-3435. doi:10.1007/ s00213-016-4381-z

59. Floam S, Simpson N, Nemeth E, Scott-Sutherland J, Gautam S, Haack M. Sleep characteristics as predictor variables of stress systems markers in insomnia disorder. J Sleep Res. 2015;24(3):296-304. doi:10.1111/jsr.12259

60. Irwin MR, Cole SW. Reciprocal regulation of the neural and innate immune systems. Nat Rev Immunol. 2011;11(9):625-632. doi:10.1038/nri3042

61. Späth-Schwalbe E, Hansen K, Schmidt F, et al. Acute effects of recombinant human interleukin- 6 on endocrine and central nervous sleep functions in healthy men. J Clin Endocrinol Metab. 1998;83 (5):1573-1579. doi:10.1210/jc.83.5.1573

62. Kubota T, Fang J, Kushikata T, Krueger JM. Interleukin-13 and transforming growth factor- $\beta 1$ inhibit spontaneous sleep in rabbits. Am J Physiol Regul Integr Comp Physiol. 2000;279:R786-R792. doi:10.1152/ajpregu.2000.279.3.r786

63. Kushikata T, Fang J, Krueger JM. Interleukin-10 inhibits spontaneous sleep in rabbits. J Interferon Cytokine Res. 1999;19(9):1025-1030. doi:10.1089/107999099313244

64. Petrov KK, Hayley A, Catchlove S, Savage K, Stough C. Is poor self-rated sleep quality associated with elevated systemic inflammation in healthy older adults? Mech Ageing Dev. 2020;192:111388. doi:10.1016/j.mad.2020.111388

65. American Psychiatric Association. Diagnostic and Statistical Manual of Mental Disorders, Text Revision. 4th ed. Washington, DC: American Psychiatric Association; 2000.

66. Vgontzas AN, Fernandez-Mendoza J, Liao D, Bixler EO. Insomnia with objective short sleep duration: the most biologically severe phenotype of the disorder. Sleep Med Rev. 2013;17(4):241-254. doi:10.1016/j.smrv.2012.09.005

67. Blair LM, Porter K, Leblebicioglu B, Christian LM. Poor sleep quality and associated inflammation predict preterm birth: heightened risk among African Americans. Sleep. 2015;38(8):1259-1267. doi: $10.5665 /$ sleep. 4904

68. Kaartinen M, Karlsson L, Paavonen EJ, et al. Maternal tiredness and cytokine concentrations in mid-pregnancy. $J$ Psychosom Res. 2019;127:109843. doi:10.1016/j.jpsychores.2019.109843

69. Zhang P, Tan CW, Chen GH, et al. Patients with chronic insomnia disorder have increased serum levels of neurofilaments, neuron-specific enolase and S100B: does organic brain damage exist? Sleep Med. 2018;48:163-171. doi:10.1016/j.sleep.2017.12.012

70. Staner L. Comorbidity of insomnia and depression. Sleep Med Rev. 2010;14(1):35-46. doi:10.1016/j.smrv.2009.09.003

71. Pilz LK, Keller LK, Lenssen D, Roenneberg T. Time to rethink sleep quality: PSQI scores reflect sleep quality on workdays. Sleep. 2018;41(5):10.1093/sleep/zsy029. doi:10.1093/sleep/zsy029

72. Fard MT, Cribb L, Nolidin K, Savage K, Wesnes K, Stough C. Is there a relationship between low-grade systemic inflammation and cognition in healthy people aged 60-75 years? Behav Brain Res. 2020;383:112502. doi:10.1016/j.bbr.2020.112502 
73. Leal G, Bramham CR, Duarte CB. Chapter 8 - BDNF and hippocampal synaptic plasticity. Vitam Horm. 2017;104:153-195. doi:10.1016/bs.vh.2016.10.004

74. Zaki N, Saleh E, Elwasify M, et al. The association of BDNF gene polymorphism with cognitive impairment in insomnia patients. Prog Neuropsychopharmacol Biol Psychiatry. 2019;88:253-264. doi:10.1016/j.pnpbp.2018.07.025

75. Wood H. Dementia: peripheral inflammation could be a prodromal indicator of dementia. Nat Rev Neurol. 2018;14:127. doi:10.1038/ nrneurol.2018.8

76. Kelly A, Lynch A, Vereker E, et al. The anti-inflammatory cytokine, interleukin (IL)-10, blocks the inhibitory effect of IL-1 $\beta$ on long term potentiation: a role for JNK. $J$ Biol Chem. 2001;276 (49):45564-45572. doi:10.1074/jbc.M108757200

77. Derecki NC, Cardani AN, Yang CH, et al. Regulation of learning and memory by meningeal immunity: a key role for IL-4. J Exp Med. 2010;207(5):1067-1080. doi:10.1084/jem.20091419
78. Richwine AF, Sparkman NL, Dilger RN, Buchanan JB, Johnson RW. Cognitive deficits in interleukin-10-deficient mice after peripheral injection of lipopolysaccharide. Brain Behav Immun. 2009;23 (6):794-802. doi:10.1016/j.bbi.2009.02.020

79. Heppner FL, Ransohoff RM, Becher B. Immune attack: the role of inflammation in Alzheimer disease. Nat Rev Neurosci. 2015;16:358-372. doi:10.1038/nrn3880

80. Chakrabarty T, Torres IJ, Bond DJ, Yatham LN. Inflammatory cytokines and cognitive functioning in early-stage bipolar I disorder. J Affect Disord. 2019;245:679-685. doi:10.1016/j.jad.2018.11.018

81. Worthen RJ, Garzon Zighelboim SS, Torres Jaramillo CS, Beurel E. Anti-inflammatory IL-10 administration rescues depression-associated learning and memory deficits in mice. $J$ Neuroinflammation. 2020;17(1):246. doi:10.1186/s12974-02001922-1

\section{Publish your work in this journal}

Nature and Science of Sleep is an international, peer-reviewed, open access journal covering all aspects of sleep science and sleep medicine, including the neurophysiology and functions of sleep, the genetics of sleep, sleep and society, biological rhythms, dreaming, sleep disorders and therapy, and strategies to optimize healthy sleep.
The manuscript management system is completely online and includes a very quick and fair peer-review system, which is all easy to use. Visit http://www.dovepress.com/testimonials.php to read real quotes from published authors. 